\title{
Dinámicas escolares asociadas a la construcción de un currículo intercultural para la paz y la justicia territorial en el Caribe colombiano, 2018-2019
}

\author{
School dynamics associated with the construction of an intercultural \\ curriculum for peace and territorial justice in the Colombian Caribbean, \\ 2018-2019
}

Dinâmica escolar associada à construção de um currículo intercultural para a paz e a justiça territorial no Caribe colombiano, 2018-2019

Recepción: 20/01/2020

Evaluación: 29/03/2020

Aceptación: 01/04/2020

Artículo de Investigación

https://doi.org/10.19053/01227238.10898

Luis Alfredo González Monroy ${ }^{1}$ https://orcid.org/0000-0001-7249-4677

Jennifer Tatiana Ortiz Segrera ${ }^{2}$ https://orcid.org/0000-0001-7732-9398

Luis David Gamarra Rosado ${ }^{3}$ https://orcid.org/0000-0003-1622-0094 Universidad del Magdalena, Colombia

\section{RESUMEN}

Objetivo: El artículo esboza una comprensión en torno a la elaboración de un currículo intercultural para comunidades educativas donde sus miembros han sido víctimas del conflicto armado colombiano. Método: Generando un diálogo de saberes y conocimientos entre investigadores y participantes, en un ejercicio de formación recíproca que llevó a reivindicar la tradición oral, que ha sido

subalternizada por los discursos escolares del orden transnacional. Estrategias: El horizonte teleológico se trazó en la concepción de hacer una intervención cooperativa, en la cual participó una universidad y una escuela pública rural con el fin de empoderar categorías como interculturalidad, paz y justicia territorial en sus miembros. Esta sinergia incentivó a la comunidad para dar un salto adelante

1 Doctor en Ciencias de la Educación de la Universidad del Magdalena-Rudecolombia. Docente Catedrático adscrito a la Facultad de Ciencias de la Educación de la Universidad del Magdalena. Miembro del grupo de investigación Calidad Educativa en un Mundo Plural (CEMPLU), categorizado en A1 por Minciencias, correo electrónico: lgonzalezm@unimagdalena.edu.co, dunkarinca@gmail.com

2 Candidata a magíster en Educación de la Universidad Sergio Arboleda. Joven investigadora e innovadora de la Universidad del Magdalena, financiada por Minciencias. Miembro del grupo de investigación Calidad Educativa en un Mundo Plural (CEMPLU), categorizado en A1 por Minciencias, correo electrónico: jenny.segrera.9426@gmail.com, jortizs@unimagdalena.edu.co

3 Candidato a magíster en Educación de la Universidad Sergio Arboleda, joven investigador de la Facultad de Ciencias de la Educación de la Universidad del Magdalena. Miembro del grupo de investigación Calidad Educativa en un Mundo Plural (CEMPLU), categorizado en A1 por Minciencias, correo electrónico: luchogama9114@gmail.com; lgamarrar@unimagdalena.edu.co 
en su nivel de conciencia de práctica docente frente al "otro". Originalidad: Esta comunicación académica sustenta la elaboración de nuevo conocimiento a partir de las voces de los participantes in situ. Conclusión: La rutina investigativa de injerencia se asume como una herramienta crítica para el cambio social, especialmente, cuando dentro de sus marcos generales los maestros aprenden a innovar y mejorar las realidades propias de una cultura o comunidad, en la expectativa de reconstruir experiencias de aprendizaje que pueden ser propuestas a partir del diseño, implementación y evaluación de acciones pedagógicas para la diversidad.

Palabras clave: Currículo intercultural, paz, justicia territorial, interculturalidad, IAC.

\section{ABSTRACT}

This article has to do with a research carried out between 2018 and 2019 in a school of a rural setting in the Caribbean, which suffered the attacks of the Colombian fratricidal conflict. This region is characterized by its agricultural vocation of banana crops for export. In this context, appears the primary school Thelma Rosa de Arévalo as a forum in which social phenomena are manifested, which the school institution begins to lead through transformation processes, using the action research methodology using the cooperative action research(-IAC) methodology. To do this, it makes a strategic alliance with the Educational Quality in a Plural World-CEMPLU group, attached to the Universidad del Magdalena.

The epistemological process faithfully examined the school dynamics of a peasant community, associated with the construction of an intercultural curriculum that promotes territorial peace and justice. To do this, it was necessary to qualify the co-researcher teachers in the episteme "interculturality" as the central axis of the study. Their experiences and knowledge were taken into account to value their teaching expertise and understanding their sufferings as victims. In the latter aspect, the accumulated pain did not allow, in the first turn of the methodological spiral, the proposed teleological transformation will be achieved. The proposed teleological transformation will be achieved, suggesting to make a second turn of the IAC loop.

Among the results it was found that the Institutional Educational Project-PEI is a technical document but it does not reflect the local reality avoiding the growth of an intercultural curriculum, since the latter requires the local educational communities and its members they should know "themselves". Without this elemental knowledge the imprint that marks the identity of people and their communities cannot be understood.

Keywords: Intercultural curriculum; peace; territorial justice; interculturality; IAC.

\section{RESUMO}

O presente artigo está vinculado a uma pesquisa realizada entre os anos 2018-2019 em uma escola localizada no território rural do Caribe, que sofreu as consequências do conflito fratricida colombiano; caracterizado por sua vocação agrícola, consistente no cultivo de banana para exportação. É neste contexto em que emerge a escola Thelma Rosa de Arévalo, como um fórum no qual se manifestam fenômenos sociais, que a instituição escolar começa a exercer liderança, mediante processos de transformação, aplicando a metodologia de Pesquisa Ação Cooperativa-IAC. Para isso, realizou uma aliança estratégica com o grupo Qualidade Educativa num mundo Plural-CEMPLU, adscrito à Universidade de Magdalena. O processo epistemológico tratou de examinar, de 
maneira fidedigna, as dinâmicas escolares de uma comunidade campesina, associadas à construção de um currículo intercultural que promova a paz e a justiça territorial. Para isso, foi necessário qualificar os professores copesquisadores na episteme da "Interculturalidade" como eixo central do estudo. Referente a eles, não se desprezou os saberes empíricos acumulados no transcurso de suas vidas, valorizando-se suas expertises docentes e compreendendo seus sofrimentos como vítimas. Neste último aspecto, a dor acumulada não permitiu que, num primeiro giro da espiral metodológica, a transformação teleológica proposta será alcançada, sugerindo-se um segundo giro do circuito da IAC. Entre os resultados temos que o Projeto Educacional Institucional-PEI é um documento técnico que não reflete a realidade local, impedindo o desenvolvimento de um currículo intercultural, posto que este último exige das comunidades educativas locais e de seus membros o conhecer-se "a si mesmos". Sem este conhecimento elementar, não se concebe as características que marcam a identidade das pessoas e de suas comunidades.

Palavras-chave: Currículo intercultural, paz, justiça territorial, interculturalidade, IAC.

\section{INTRODUCCIÓN}

El desarrollo del proceso investigativo tuvo, como punto de partida, la premisa de entender que a los maestros les corresponde asumir actitudes favorables frente a la comprensión de la diversidad humana, manifestada en las escuelas donde ejercen su quehacer pedagógico. En esta comunicación académica se desarrolla un ejercicio de sensibilización frente a los problemas socioculturales que padecen las víctimas del conflicto colombiano. Con el acuerdo de paz, firmado por el Estado y las Fuerzas Armadas Revolucionarias de Colombia-FARC-EP, se abrió un espacio de esperanza para quienes habían sufrido desplazamiento forzado, usurpación de tierras y pérdidas de allegados. Se viene observando en los distintos espacios liberados de guerra, un nuevo fenómeno al que se le ha denominado "retorno" ${ }^{4}$. En este sentido, se hace indispensable trazar un nuevo horizonte como oportunidad para resignificar categorías como paz, justicia y territorio. Se presenta entonces un escenario en el cual, más allá de tolerar al otro en su diferencia, se ha de cristalizar el hecho inalienable de comprenderlo. Lo anterior lleva a José Ignacio Bolaños a proponer a la escuela como un entorno de comprensión, en los siguientes términos:

La dejación de las armas con el grupo armado más grande del país se ha logrado $y$ ahora se debe educar para la paz y para armonizar la diferencia, aprendiendo a aceptar las otredades como la diversidad étnica, religiosa, política, al exparamilitar, al exguerrillero... La comprensión de los nuevos otros es un proceso extenso. No es tarde para empezar y se espera que ello sea posible. ${ }^{5}$

$4 \quad$ Cfr. Luis Alfredo González Monroy, "Escuelas del retorno después del desplazamiento forzado en Colombia. Significados y sentidos dialógicos de un currículo intercultural" (tesis de doctorado en Ciencias de la Educación-RUDECOLOMBIA, Universidad del Magdalena, 2015), 15.

5 José Ignacio Bolaños Motta, "Aulas multiculturales y aulas de paz. Dos propuestas para un país en postconflicto", Revista Historia de la Educación Latinoamericana vol. 20, n. ${ }^{\circ} 31$ (2018): 97-98. 
La escuela no ha sido ajena a este hecho y corresponde a sus actores delinear la formación de los niños, niñas y jóvenes, tarea asumida con una responsabilidad que va más allá de lo laboral, vislumbrada como un compromiso con la sociedad y la comunidad donde se ejerce magisterio, siendo además conscientes de que se ha de trabajar para formar un alumnado y una comunidad educativa que transcienda en el desarrollo de la sociedad y el país.

Algunas comunidades no han logrado comprender que - si bien la plantea un Estado que se ha distinguido principalmente por su indolencia - esta es sin duda una nueva oportunidad. En este aspecto es necesario traer a cuento el caso de la comunidad perteneciente a la Institución Educativa Departamental Thelma Rosa Arévalo, ubicada en la población de Varela, municipio de Zona Bananera, departamento de Magdalena, que ha logrado apreciar la necesidad de establecer procesos en la escuela que les han permitido fortalecer la identidad, la pertenencia hacia la comunidad y la institución, pero encapsulándose en posturas eurocéntricas, hegemónicas y homogeneizantes, en una actitud del laissez-faire ${ }^{6}$.

En consecuencia, surge la inaplazable tarea de jalonar procesos educativos que involucren a todos los miembros comunitarios, teniendo como referente epistémico su acervo sociocultural para elaborar planes de vida que coadyuven a cristalizar sociedades en el buen vivir en tranquilidad. Corresponde entonces al estamento docente liderar transformaciones generadoras de impacto duradero en "el tiempo y el espacio curricularizado, pues se toman otros ritmos en enlace con las necesidades de sus estudiantes"7. Por ello, es necesario un acompañamiento con miras a resignificar el currículo de manera consensuada desde las voces de los miembros y que aporte elocuentemente a la construcción de una escuela de paz. Se hizo necesario, entonces, hacer el abordaje científico en el establecimiento mencionado, a través de la metodología Investigación Acción Cooperativa, donde actuaron científicos y maestros de manera mancomunada, al mismo nivel, en una reciprocidad de aprendizajes de sentido.

La preocupación teleológica del estudio consistió en develar las dinámicas escolares de una comunidad agrícola asociada a la construcción de un currículo intercultural para la paz y la justicia territorial, en un escenario cuya vocación laboral está soportada en el cultivo del banano, que además ha tenido como característica el haber sufrido los rigores del conflicto armado colombiano.

\section{Constructo epistemológico}

En las dinámicas de la escuela se evidencian las realidades sociales y cosmogónicas de su entorno. Se asume el aula como el espacio donde estudiantes, padres de familia y maestros, esperan ser escuchados, con la esperanza de ser reconocidos en sus individualidades y en sus roles como miembros de un colec-

6 Dejar hacer o dejar pasar desde el ámbito administrativo [nuestra traducción].

7 Lilia Cañón Flórez, Myriam Monroy González y Javier Salcedo Casallas, "Experiencias pedagógicas y voz multicultural. Pasos y palabras en historias de vida de maestra afrodescendiente". Revista Historia de la Educación Latinoamericana vol. 18, n. 27 (2016): 177. 
tivo social; sin embargo, el ambiente escolar está plagado de dogmas y doctrinas traídas de otros países, puesto que se concibe la educación como una inversión, llevándola a un estado de industrialización donde sus beneficiarios se asumen como consumidores y no como seres humanos.

La anterior situación soporta propuestas elaboradas desde la emancipación académica, en las cuales se estrechen las brechas sociales mediante estrategias que articulen los procesos educativos, en una lógica donde el currículo escolar pendule entre lo productivo y lo reproductivo de la sociedad que se pretende formar, siempre bajo el presupuesto de los saberes propios y del territorio que llama al arraigo, sin desconocer el conocimiento científico ofrecido en el plantel educativo. En esta coyuntura, "es necesario poner de manifiesto que toda persona está en capacidad de desarrollar y perfeccionar sus capacidades durante su existencia, además de entender la complejidad que conforman la educación en saberes, instituciones y sujetos" ${ }^{8}$.

De esta manera, cuando los miembros de una comunidad detienen sus quehaceres diarios para reflexionar acerca de ellos mismos y de los otros, aquellos que los rodean, con los que se comparte vida, es cuando se hace consciencia que se es diferente. Se es parte de una comunidad donde todos se toleran, emergiendo entonces el concepto de multiculturalidad ${ }^{9}$. Pero la calidad de vida va más allá de esa tolerancia, se encuentra inmersa en el convivir con el otro, comprendiéndolo. Esta reflexión lleva a constituir la categoría interculturalidad ${ }^{10}$, que para la escuela da apertura a condiciones de equidad, justicia social y reconocimiento de la diversidad cultural que en ella subyace.

El proceso investigativo tuvo como pistas epistemológicas, seis trabajos empíricos soportados en la categoría interculturalidad, entendida como cimiento central del estudio realizado. En este orden de ideas, se inicia con el estudio desarrollado por Lehmann, quien realizó una revisión sistemática documental en cinco universidades mexicanas; su aporte científico aprehendido fue el ensamblaje epistémico de las categorías ethos e interculturalidad, y el consecuente debate académico que suscitó entre los años 2006 y 2009. Al abordar la categoría educación intercultural en una línea del tiempo, Lehmann manifiesta:

[...] la educación intercultural ha tenido varios propósitos, entre ellos concientizar a los portadores de las culturas dominantes sobre la existencia y el valor de otras culturas y, viceversa [...] Se ha tratado de alcanzar una situación de respeto mutuo $y$ de superación de las condiciones de exclusión social en la sociedad como un todo, no solo entre los pueblos indigenas. ${ }^{11}$

\footnotetext{
$8 \quad$ Fredy Luis Pérez Dávila, "Políticas educativas en Colombia: en busca de la calidad”, Actualidades Pedagógicas n. ${ }^{\circ} 71$ (2018): 195.

9 Fidel Tubino, "El interculturalismo y los Estados nacionales" (conferencia magistral, Foro latinoamericano sobre interculturalidad, ciudadanía y educación, FLAPE, Cuetzalán, México, noviembre de 2004).

10 Ibid. 1-15.

11 David Lehmann, "Convergencias y divergencias en la educación superior intercultural en México", Revista Mexicana de Ciencias Políticas y Sociales vol. LX, n. ${ }^{\circ} 223$ (2015): 136.
} 
Se observa, desde la perspectiva de Lehmann, que la interculturalidad viene adquiriendo un posicionamiento en el discurso latinoamericano, no como una bandera ondeada por la cosmogonía de los pueblos originarios, sino como una nueva alternativa para reivindicar la dignidad de las personas en un mundo para todos.

En segundo lugar, Catherine Walsh en la introducción que hace en el tomo 1 del libro: "Pedagogías decoloniales" concibe la interculturalidad como detonante de la decolonialidad que abre caminos y condiciones radicalmente a "otros" en pensamiento, resurgimiento, levantamiento y edificación ${ }^{12}$. Lo anterior a través de prácticas entendidas pedagógicamente que, a la vez, hacen cuestionar y desafiar la razón única de la modernidad occidental y el poder colonial aún presente, desenganchándose de ellos. Walsh concluye que la colonización cultural intentó amputar nuestra voluntad de cambiar el mundo, razón por la cual el que descoloniza su pensamiento debe actuar como un sembrador que siembra semillas en forma de ideas y premisas preñadas de indignación y esperanza.

Son los indignados los que más rápidamente comprenderán la necesidad de realizar la decolonialidad de su pensamiento, si quieren contribuir a la decolonialidad de la historia, la educación, la economía, el Estado, la agricultura, la salud o cualquier otro campo de interés de la sociedad. Con respecto a la educación, De Souza Silva afirma:

La educación no es un fin en sí misma, es un "proceso-medio" - de intervención en la formación de ciudadanos y ciudadanas - para reproducir una sociedad, cuando su orden es relevante para todos, o para transformarla en otra sociedad, cuando su orden es violento, desigual e injusto, a partir de otra filosofía de vida, otra pedagogía y otra educación que incorpore dicha filosofía y pedagogía. ${ }^{13}$

En la cita anterior se da un giro a la categoría educación intercultural, al asumirla como una opción para hacer crítica a los modelos establecidos en las instituciones del Estado, entre ellos la misma escuela. Es decir, se empodera la interculturalidad como una filosofía de vida, pero también como pedagogía que es capaz de transformar sociedad.

En un tercer momento se estimó la reflexión realizada por Elizabeth Castillo y Sandra Guido a la que titularon "La interculturalidad: ¿Principio o fin de la utopía?", en la cual las autoras ofrecen una mirada panorámica en torno a los asuntos del poder, la exclusión y la subalternidad, desde dos perspectivas específicas. De una parte, describen el recorrido que esta interculturalidad al constituirse como objeto generador de nuevos conceptos y teoría, tejidos alrededor de saberes locales y globales. Y de otra, el destello que la interculturalidad ha

12 Walsh Catherine. "Pedagogías decoloniales. Prácticas insurgentes de resistir, (re) existir y (re)vivir”. Tomo 1. (Quito: Editorial Abya Yala, 2018), 12.

13 José de Souza Silva, "La pedagogía de la felicidad en una educación para la vida”, en Pedagogías decoloniales: Prácticas insurgentes de resistir, (re)existir y (re)vivir, tomo I ed. Catherine Walsh (Quito: Ediciones Abya Yala, 2018), 476. 
venido adquiriendo en Colombia, a pesar de haber sido invisibilizada en comunidades que presentan serios signos de diferencia y diversidad.

La tendencia es que lo intercultural "destaca" la relación que deben establecer las minorías con la mayoría, en el marco del reconocimiento de sus derechos y la formulación de políticas educativas. Ahora bien, este énfasis olvida que la llamada sociedad mayoritaria es la que más urgencia de interculturalidad tiene, en la medida en que es en su seno donde se producen y reproducen las formas de discriminación y exclusión hacia las llamadas "minorías". ${ }^{14}$

En cuarto lugar, se evoca a Emilce Sánchez, quien logró demostrar que la acción de educar para enseñar y aprender ha de estar contextualizada dentro del ámbito de la cultura que se pretende abordar ${ }^{15}$. En este sentido, las experiencias que emergen de la doxa en el contacto real con las comunidades, siempre han de estar en sincronía con el desarrollo pleno de lo que se concibe como interculturalidad. Esta última episteme permite el reconocimiento de la identidad territorial, y para ello, el diálogo recíproco es jalonador de principios de la autonomía comunitaria que impulsa las culturas originarias. Lo dicho lleva a pensar en la cualificación de los maestros en etnoeducación, para fortalecer las culturas originarias subyacentes en los escenarios educativos, rechazando los discursos hegemónicos de la cultura colonizadora. De igual manera, cobra fuerza el reconocer otros modelos pedagógicos para acometer la cultura y sus saberes heredados. Sánchez propone en este aspecto:

La expresión actual de la visibilización, el fortalecimiento cultural, la aceptación de la diversidad en el marco amplio de la cultura, apenas comienza. Estos ideales presionan a la escuela en zonas de contacto intercultural a transformarse, abrirse a nuevas posibilidades de desarrollo no solo académico, sino también en el sistema de creencias y de valores que posibiliten la apertura a una nueva pedagogía -etnopedagogía - que interprete los contenidos de la cultura y sea capaz de armonizarlos tanto con los planteamientos de la pedagogía occidental como con los intereses de los actores originarios de una cultura. ${ }^{16}$

En quinto lugar, surge Inés Olivera Rodríguez con una propuesta denominada "Buen vivir", en la cual presenta la educación formal como ese espacio fundamental para el desarrollo del ser y el aporte para la construcción de vidas dignas. Mirada crítica que devela la función social de la educación en relación con los saberes ancestrales.

14 Elizabeth Castillo Guzmán y Sandra Patricia Guido Guevara, “La interculturalidad: ¿principio o fin de la utopía?”, Revista Colombiana de Educación, n. ${ }^{\circ} 69$ (2015): 39-40.

15 Emilce Beatriz Sánchez Castellón, “Etnoeducación y prácticas interculturales de saberes otros”, Utopía y Praxis Latinoamericana vol. 23, n. ${ }^{\circ} 83$ (2018).

16 Ibid., 22. 
Las habilidades y competencias permanecen vinculadas con la capacidad de identificar saberes locales y hacerlos dialogar con los saberes occidentales, con la habilidad de comunicarse en lenguas indígenas para posibilitar el acceso a saberes locales, $y$ con la capacidad de realizar trabajos colaborativos y promover la acción colectiva desde una comprensión que cambia la idea de "extensión universitaria" por la de "vinculación con la comunidad", y que pasa de la investigación como ejercicio de extracción a la investigación vinculada basada en el diálogo de saberes. ${ }^{17}$

Finalmente, la categoría intercultural llega a un híbrido con el currículo escolar; Luis Alfredo González e Iván Sánchez le dan el sentido de herramienta que ha de ser empleada por las nuevas generaciones como proyecto de reivindicación de su cultura legada, para luego revalorizarla y transmitirla a su descendencia.

En una búsqueda incesante, en la cual esta última pueda gozar y tener opción de participar en una sociedad del bienestar, activa, crítica, justa e intercultural, que coadyuva en la construcción de un proyecto de vida colectivo, donde se evidencien sus capacidades para sobreponerse a sus tropiezos, logrando realizarse como personas verdaderamente humanas, ricas en culturas y en sueños. ${ }^{18}$

Por otra parte, la investigación se soportó en cuatro categorías epistémicas: currículo, interculturalidad, etnoeducación y escuela para la paz. Ámbitos que en la educación actual marcan el derrotero de interacción entre el pensamiento eurocéntrico y la cosmovisión de los pueblos originarios. En este orden de ideas, al afrontar la categoría currículo se encuentra que es un término educativo al que comúnmente se le atribuyen diversos significados. Su finalidad es transformar la educación adoptando distintas posiciones ideológicas, filosóficas, epistemológicas, pedagógicas y socioculturales, integrando de esta manera un concepto oportuno que ayude a mejorar unas necesidades y exigencias propias de un contexto. La Ley 115 de 1994 lo define como el:

Conjunto de criterios, planes de estudio, programas, metodologías y procesos que contribuyen a la formación integral y a la configuración de la identidad cultural nacional, regional y local, incluyendo también los recursos humanos, académicos y físicos para poner en práctica las políticas y llevar a cabo el proyecto educativo institucional. $^{19}$

Desde una mirada académica, el currículo es la manera como los procesos de enseñanza y aprendizaje se organizan para orientar y buscar el mejoramiento de una formación pertinente para los estudiantes. El currículo ha de promover

17 Inés Olivera Rodríguez, "Las potencialidades del proyecto educativo de la Universidad Veracruzana Intercultural: una crítica al desarrollo desde la noción del Buen vivir", Revista de la Educación Superior vol. 46, n. ${ }^{\circ} 181$ (2017): 35.

18 Luis Alfredo González Monroy e Iván Manuel Sánchez Fontalvo, Interpelaciones interculturales en el currículo de las escuelas del retorno. Referentes para vislumbrar el posconflicto en Colombia (Santa Marta, Colombia: Editorial Unimagdalena, 2016), 65.

19 Ley 115/1994, 8 de febrero, Ley General de la Educación (Diario Oficial n. ${ }^{\circ} 41.214$ ) http://www.secretariasenado.gov.co/senado/ basedoc/ley_0115_1994.html (9 de abril de 2020). 
la equidad a partir de las condiciones en que se desarrolle la escuela. Conjuntamente, sirve para guiar una institución educativa, potenciando la labor del docente y asimismo el proceso de aprendizaje del estudiante. Del mismo modo, este se debe precisar con unos objetivos institucionales para cumplir con su finalidad.

El currículo se expresa a partir de unos referentes que ayudan a los actores educativos a direccionar el fortalecimiento de valores, competencias y capacidades del individuo. Éste ha de tener en cuenta las necesidades del contexto escolar para articular todos los procesos académicos, como también los elementos educativos de sus participantes.

\begin{abstract}
Existe un importante consenso entre los teóricos del currículo cuando afirman que éste es una selección cultural o de otro modo que es una organización selectiva de la cultura social para ser enseñada y aprendida en las escuelas. Pero el disenso radica en cómo entender la cultura social y sus elementos más relevantes. Por nuestra parte desde una perspectiva integradora, no ecléctica como ya hemos indicado, consideramos que la cultura social indica las capacidades, los valores, los contenidos y los métodos-procedimientos que utiliza una sociedad determinada, en su dimensión diacrónica y sincrónica. Por ello, los elementos básicos de la cultura social son las capacidades, los valores, los contenidos y los métodos-procedimientos. $^{20}$
\end{abstract}

Desde esta perspectiva, el currículo como práctica social y humana involucra un momento eficiente en la organización y configuración permanente de una cultura que está establecida por las características específicas de su ámbito social. Es por ello que debe procurar una mejora educativa y una transformación de la sociedad, originando pedagogías alternativas que propicien una continua interlocución con las comunidades para incorporar sus propuestas en la construcción de la $\mathrm{paz}^{21}$, a través de los intereses y necesidades propias del mismo entorno sociocultural. En la actualidad, la realidad escolar demanda una resignificación curricular en perspectiva de los procesos teóricos y prácticos, en relación con las acciones de la labor formativa.

En este sentido, el currículo desde su concepción garantiza los procesos académicos, dando respuesta a las prácticas socioculturales para así mejorar y afianzar los principios de una cultura y, del mismo modo, el de las instituciones educativas. Igualmente, el currículo ha de superar modelos tradicionalistas de enseñanza para innovar y mejorar las realidades propias de una cultura o comunidad, en la expectativa de reconstruir experiencias de aprendizaje que pueden ser propuestas a partir del diseño, implementación y evaluación de las acciones pedagógicas, enmarcadas en la reflexión y selección cultural que permitan facilitar las intenciones del enfoque curricular.

20 Juan José Fonseca Pérez y Michel Enrique Gamboa Graus, "Aspectos teóricos sobre el diseño curricular y sus particularidades en las Ciencias, Boletín Virtual vol. 6, n. ${ }^{\circ} 3$ (2017): 88-89.

21 Cfr. Celina Triviño y Luisa Amézquita, "Reflexiones desde la universidad sobre la educación en derechos humanos y para la paz", Revista Historia de la Educación Latinoamericana vol. 20, n. ${ }^{\circ} 31$ (2018): 114. 
De otra parte, la interculturalidad hace mención a la construcción de las distintas culturas con relación a la interrelación, correspondencia y tolerancia. De igual manera, significa establecer colaboración entre las comunidades y etnias, teniendo como finalidad preservar y mantener sus costumbres y tradiciones dentro de los parámetros de la sociedad. La interculturalidad destaca las particularidades en común con los demás, sin tener en cuenta el ámbito personal. Para Iván Sánchez, una persona intercultural, en particular,

[...] es alguien que es curioso en vez de temeroso con respecto a otras culturas y personas; alguien, que está abierto a aprender de otros estilos de vida y dispuesto a considerar cómo se ven las cosas desde el punto de vista de otra gente en vez de asumir como superior su perspectiva o modo de vida heredado; alguien que se siente cómodo interactuando con personas de diferentes ambientes, etc. ${ }^{22}$

La interculturalidad se da hoy en todos los contextos, territorios, instituciones educativas, debido a que se fundamenta en la formación de las personas para obtener las habilidades y destrezas que permiten relacionarse con los diferentes individuos, colectivo de personas y con la sociedad, dentro de parámetros de respeto, capacidad de diálogo y la coexistencia de la valoración sociocultural, fomentando los modos y estilos de vida de las comunidades. Concluye Gavina Córdova:

Como concepto y práctica significa "entre culturas" pero no meramente como contacto, sino en un intercambio permanente de relación, comunicación y aprendizaje entre personas, grupos, conocimientos, valores y tradiciones distintas que se orientan a generar, construir y propiciar un respeto mutuo para desarrollar sus capacidades por encima de las diferencias culturales y sociales. ${ }^{23}$

Hoy el mundo se encuentra abierto a escuchar y aceptar cualquier aporte que pueda favorecer a la cultura, desde cualquier punto de vista. Nos encontramos inmersos en una sociedad costumbrista, con pocos líderes formados en la emancipación y el espíritu crítico como eje transversal de la interculturalidad, en el ideal de formar personas con sentido social. En este aspecto, Edison Higuera y Néstor Castillo piensan que esta categoría:

[...] además, exige la práctica de un conjunto de valores éticos frente a las diferencias culturales presentes en el territorio de un mismo Estado: reconocimiento, comprensión, respeto, comunicación y actitudes favorables; valores que no se desarrollan por generación espontánea, sino que deben ser cultivados a lo largo de la vida. ${ }^{24}$

22 Iván Manuel Sánchez Fontalvo, La interculturalidad desde la perspectiva de la inclusión socioeducativa (Santa Marta: Editorial Unimagdalena, 2017), 76.

23 Gavina Córdova, “Diálogo sobre interculturalidad”, ISEES: Inclusión Social y Equidad en la Educación Superior n. 7 (2010): 108.

24 Édison Francisco Higuera Aguirre y Néstor Castillo Mantuano, "La interculturalidad como desafío para la educación ecuatoriana", Sophia: Colección de Filosofía de la Educación n. ${ }^{\circ} 18$ (2015): 151. 
Pero también la interculturalidad a través de la dialogicidad crea momentos donde las distintas personas dinamizan sus habilidades desde la iniciativa propia, permitiendo un desarrollo autónomo con base en el empoderamiento de las realidades locales. Situación que nos lleva a lograr un avance enfocado en la participación, el diálogo, el intercambio de conocimiento y el debate. Todos aspectos que permiten generar acuerdos para desplegar con éxito el trabajo en equipo. Asimismo, abarca de manera generalizad la participación de toda una "sociedad", permitiendo el descubrimiento y los aportes que pueden hacerse entre culturas, sin generar variaciones o alteraciones de las mismas.

Por su parte, la etnoeducación se viene entendiendo como el conjunto de procesos comunitarios, que mejoran el dinamismo cultural de las propias comunidades. Su objetivo se plasma en mantener y potencializar entre otras perspectivas, la identidad, las tradiciones y costumbres, desde acciones educativas, garantizando la conservación de las culturas en sus territorios de vida. Del mismo modo, la Ley 115 de 1994, la entiende como:

[...] educación para grupos étnicos la que se ofrece a grupos o comunidades que integran la nacionalidad y que poseen una cultura, una lengua, unas tradiciones y unos fueros propios y autóctonos Esta educación debe estar ligada al ambiente, al proceso productivo, al proceso social y cultural, con el debido respeto de sus creencias y tradiciones. ${ }^{25}$

Fabio García argumenta que la etnoeducación es un discurso y un ejercicio identitario, que se proyecta sobre la epistemología del conocimiento que soporta el tratamiento de las relaciones sociales, políticas y económicas, en un contexto nacional y mundial en el que el discurso y las prácticas se plantean desarticular y desinstalar postulaciones hegemónicas para situar en un plano valorativo la pertenencia étnica, la diferencia de identidad y el desconocimiento de cualquier valor dado a la raza y al racismo en tal orden de relaciones ${ }^{26}$.

Por ello la etnoeducación establece el desarrollo de las relaciones sociales con quienes integran un grupo o comunidad cultural, formando conocimientos, desarrollando competencias según sus cualidades, permitiendo la realización pertinente del desarrollo comunitario y así concebirse identitariamente frente a las demás culturas. En el contexto colombiano, la etnoeducación ha tenido como referente las poblaciones indígenas y afro, con un constructo socio-histórico, cultural, cosmogónico y educativo, con matices de emancipación y posturas críticas. Su carácter reivindicativo conlleva a rupturas con modos de ver el mundo heredados del colonialismo euro-norteamericano, en una verticalidad que abre brechas entre iguales. Yeison Meneses concibe la escuela etnoeducativa en estrecha relación con las comunidades, ambas:

25 Ley 115/1994.

26 Fabio Alberto García Araque, "La etnoeducación como elemento fundamental en las comunidades afrocolombianas", Diálogos sobre Educación vol. 8, n. ${ }^{\circ} 15$ (2017): 19. 
[...] caminan de manera dialógica: hay una relación horizontal que permite a la comunidad pensar y construir el tipo de educación deseado. La participación activa de la comunidad y todos los sujetos escolares afectan la organización escolar, el Proyecto Educativo Institucional, el currículo, planes de estudios. Es un una conversación entre la cultura experiencial y la cultura académica. ${ }^{27}$

En este sentido, la etnoeducación tiene relevancia para los distintos grupos culturalmente indígenas, rom y afrodescendientes, ayudándoles a restablecer sus valores y principios autónomos, siendo así protagonistas del proceso de enseñanza-aprendizaje. Asimismo, estos actores de los contextos in situ llegan a aprender desde de sus propias tradiciones y costumbres, a concientizarse de poseer una identidad cultural propia y sui generis. Por lo tanto, la persona que se prepara como etnoeducador ha de verse a sí misma como facilitadora del diálogo de saberes, conjugando sus experiencias comunitarias con la praxis académica adquirida, como forma otra de complementariedad en la construcción de saber, elaboración del ser y transformación social ${ }^{28}$.

De otra parte, cuando se evoca la escuela para la paz la educación es dimensiona como pilar de la sociedad, ella se vislumbra como el espacio de reflexión, el diálogo, la colaboración y demás valores fundamentales para la formación de las personas. En este sentido, la escuela ya no se puede considerar como el lugar donde se transmiten conocimientos, su vocación va dirigida en recrear en sus distintos estamentos prácticas del buen vivir en tranquilidad. Por ello, en una sociedad constituida por unas comunidades culturalmente diferentes, en una geografía en la cual el conflicto fratricida ha hecho nido, corresponde a la institución escolar promover entre sus miembros distintas alternativas pedagógicas para que se cristalice la paz como cultura de armonía en la cual se beneficia a la sociedad en general.

Entonces, es necesario darles un espacio a los saberes locales que emergen en las comunidades, puesto que éstos contienen los valores familiares que se legan a los hijos y nietos, entre ellos, pautas de convivencia y trato con los otros.

Es importante que los centros educativos indispensablemente sean escuelas, colegios, universidades $u$ otras entidades relacionadas con el aprendizaje, sean conscientes de la carga cultural y la influencia de la familia a la hora de los aprendizajes no solo conceptuales sino éticos, morales y religiosos, esto es indispensable para poder avanzar en las campañas o eventos que favorecen la cultura de paz y poder dar una orientación desde las instituciones más enfocada en aquellos factores de riesgo arraigados a la violencia. ${ }^{29}$

27 Yeison Meneses Copete, "La etnoeducación afrocolombiana: conceptos, trabas, patriarcado y sexismo. A propósito de los 20 años de la Ley General de Educación 115 de 1994”, Revista Historia de la Educación Latinoamericana vol. 18, n. 27 (2016): 54.

28 Ibid., 52.

29 Vivian Arenas, Karina Mora Wilches y Lisney Medina Mendoza, "Familia y escuela como entidades fundamentales en la construcción de paz", AIBI revista de investigación, administración e ingeniería vol. 7, n.o 51 (2019): 26. 
La anterior postura lleva a pensar que la escuela es una unidad social fundamental, en la cual los procesos llevados a su interior han de favorecer, desde cualquier perspectiva, el pensamiento crítico. De esta manera, la práctica educativa permite la interrelación de todos sus protagonistas, facilitando el diálogo generador de coexistencia en tranquilidad que logre transformar las comunidades y potencialice las identidades propias.

Necesitamos una escuela que enseñe también a convivir en un entorno de paz, entendida ésta en su sentido positivo, es decir, no sólo como una ausencia de guerra o de violencia directa, sino como un estado y un proceso activo de bienestar $y$ seguridad donde los derechos humanos son respetados y las necesidades humanas básicas son satisfechas; y como antes se dijo, uno de los elementos principales de esa cultura de paz lo constituye precisamente la gestión pacífica de los conflictos, de ahí la importancia de la prevención. ${ }^{30}$

Por lo tanto, la escuela como laboratorio de paz insinúa en sus miembros la toma de decisiones para aplacar cualquier situación de conflicto que se dé en su entorno; sin embargo, es preciso señalar que cuando se habla de guerra, se está haciendo referencia a una problemática mayor, con pocas posibilidades de finiquitarse en un futuro cercano. Si bien es cierto que desde esta se vulneran los derechos humanos y fundamentales de las personas que son victimizadas, la sociedad colombiana aún no está completamente preparada para afrontar las secuelas del conflicto, si se encuentra dando sus primeros pasos para realizarlo y en ello la escuela adquiere gran importancia.

Sostenemos que la educación para la paz debe investigar acerca de la forma de acompañar a cada persona en la realización de análisis de su realidad y del mundo en el que vive, promoviendo reflexiones, pensamientos y actividades que han de poder concretarse en la vida diaria, generando un conocimiento aplicable y útil que nos facilite la posibilidad de encontrar, cada día, nuevas y mejores formas de convivencia, desarrollo personal y construcción comunitaria. ${ }^{31}$

Para sintetizar, la escuela podría concebirse como escenario de transformación social donde se facilita la construcción de una cultura de paz. En ella se hace fundamental la cooperación de todos los miembros de la comunidad educativa, en la cual no se tienen en cuenta las diferencias sociales, culturales, políticas, económicas o de otra índole, puesto que se hace un constructo por el respeto y tolerancia de la diversidad, reconociendo así los intereses de cada persona y sobre todo de la comunidad en general.

Asimismo, cuando De Oña y García se refieren a la cultura de la paz, la vislumbran como un proceso inacabado en continua construcción, donde ámbitos como

30 Elizabeth Tostado Reyes y Saúl-Alejandro García, "Convivencia con conflicto y sin violencia en la escuela: la prevención como estrategia de paz”, Ra Ximhai vol. 11, n. ${ }^{\circ} 1$ (2015): 29.

31 José Manuel de Oña Cots y Emilio Andrés García Gálvez, "Proyecto Escuela: Espacio de Paz. Reflexiones sobre una Experiencia en un Centro Educativo", REICE. Revista Iberoamericana sobre Calidad, Eficacia y Cambio en Educación vol. 14, n. ${ }^{2}$ (2016): 129. 
los valores, solidaridad, paciencia y respeto, inspiran aprendizajes que ofrecen a las personas posibilidades de satisfacer sus necesidades en desarrollo de las dinámicas de la vida del día a día en el disfrute de una existencia en tranquilidad $y$ en libertad ${ }^{32}$.

\section{Metodología}

La metodología asumida en el estudio fue la Investigación Acción Cooperativa (IAC), la cual permitió trabajar mancomunadamente con las comunidades implicadas en la problemática planteada, cuya necesidad consistió en preguntarse cuáles son las dinámicas escolares de una comunidad agrícola asociada a la construcción de un currículo intercultural para la paz y la justicia territorial, en concordancia con las demandas socioculturales y políticas, pasando por el reconocimiento de la historia e incorporándose emergente y transversalmente en el plan de vida de la población local donde tiene inherencia la escuela.

Por lo tanto, la Investigación Acción Cooperativa, según Margarita Bartolomé, es aquel tipo de investigación-acción que se da cuando algunos miembros del personal de dos o más instituciones, generalmente una de ellas orientada a la producción de la investigación científica o a la formación de profesionales (Universidad del Magdalena) y siendo la otra una escuela o una institución (Institución Educativa Departamental Thelma Rosa de Arévalo) en la que trabajan esos profesionales a los que se pretende formar, deciden agruparse para resolver juntos problemas que atañen a la práctica profesional de estos últimos, vinculando los procesos de investigación con los procesos de innovación y con el desarrollo y formación profesional ${ }^{33}$.

33 Margarita Bartolomé, "La investigación cooperativa", en Problemas y métodos de investigación en educación personalizada, coord. Víctor García Hoz (Madrid: Rialp, 1994), 385. 


\section{Gráfico 1. Fases de la Investigación Acción Cooperativa}

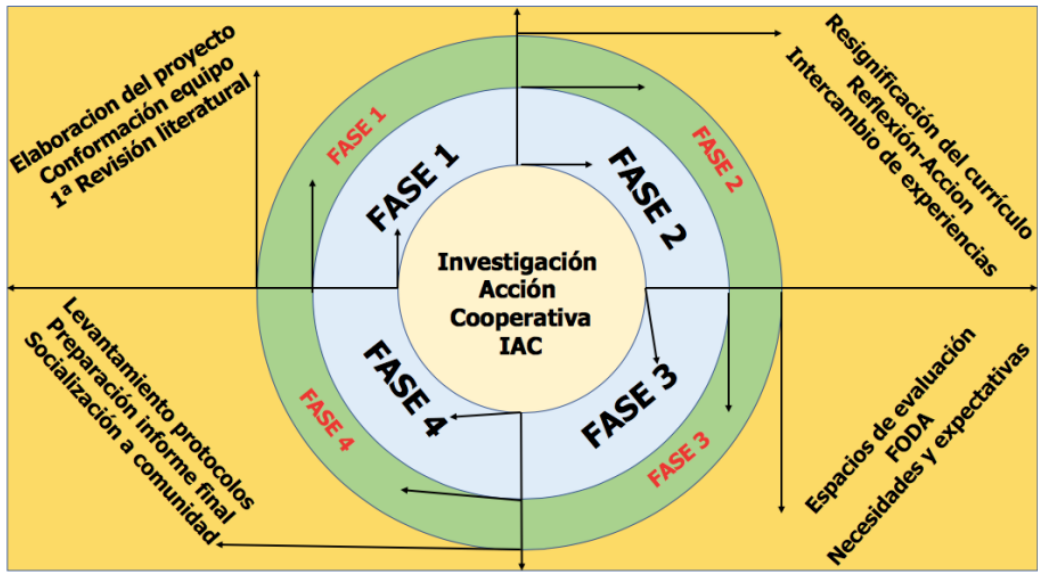

Fuente: Adaptado de Oja y Pine (1983) ${ }^{34}$.

La metodología se desarrolló a partir de cuatro fases que conforman el diseño metodológico, que pueden ser establecidas en el proceso:

- 1. ${ }^{\text {a }}$ fase: Revisión de la literatura, lanzamiento del proyecto en la comunidad del corregimiento de Varela (Magdalena), constitución de los grupos de trabajo con los distintos miembros que representan los estamentos de la comunidad educativa de la Institución Thelma Rosa Arévalo, reconocimiento de los pilares y estrategias que orientan el quehacer educativo y las realidades vivenciadas en la comunidad, ajuste del Proyecto Educativo Institucional(PEI) a las necesidades, intereses y expectativas de la comunidad.

- 2. ase: Resignificación del currículo. A través de encuentros de inmersión empíricos para la construcción de las categorías que componen el currículo intercultural para la paz y justicia territorial. Proceso de reflexión-acción participante con los diferentes equipos, lo cual se constituye en plataforma de intercambio de experiencias.

- 3. a fase: Con el ánimo de adaptar la resignificación curricular a las necesidades detectadas, se posibilitaron espacios de evaluación con miras a detectar entre los actores socioeducativos de la comunidad y el equipo académico de la Universidad del Magdalena las posibles fortalezas y debilidades del proceso y las oportunidades y amenazas que puede tener, de cara a adaptar sus actividades para que se ajusten a las necesidades y expectativas del entorno donde está inmersa la institución educativa.

- 4..$^{\mathrm{a}}$ fase: Se constató la necesidad de mantener un rigor metodológico a lo largo de todo el proceso que implicó, por una parte, la realización de protocolos que permitieron garantizar el conocimiento y fundamentación

34 Sharon Nodie Oja y Gerald J. Pine, Collaborative Action Research: A Two Year Study of Teachers' Stages of Development and School Contexts. Executive Summary (New Hampshire University, 1983). 
de los procesos seguidos, los cuales servirán como punto de partida de nuevos proyectos y constituirán el inicio de un repositorio documental alojado en ambas instituciones: Universidad del Magdalena y IED Thelma Rosa Arévalo. De igual manera, se hizo devolución a las comunidades participantes del conocimiento que de ellas se recogió y sistematizó ${ }^{35}$.

\section{Resultados}

El currículo escolar de la Institución Educativa Departamental Thelma Rosa Arévalo, no se distancia de los acontecimientos socioculturales y cosmogónicos de su comunidad educativa. Sus miembros participan en la celebración de las fiestas patronales en honor de San Martín de Loba, en eventos como bautizos y primeras comuniones, el día 11 de noviembre de cada año. Devoción que gira en torno de las buenas ventas, el amor, el dinero. Igualmente, las instalaciones de la institución son utilizadas por grupos religiosos protestantes para realizar sus cultos. El PEI hace referencia a este respecto en los siguientes términos: "Tratar con respeto, imparcialidad, rectitud y justicia a los estudiantes, sin ninguna clase de preferencias ni distingo, que permitan el ejercicio y la libertad de culto y de creencias religiosas" 36 .

De otra parte, el currículo institucional ofrece en su parte técnica cuatro modalidades: gestión agro-empresarial, salud ocupacional, legislación laboral y práctica empresarial. Cada una de estas modalidades tiene a su cargo la organización de los proyectos que permitan la aplicabilidad de los conocimientos adquiridos en competencias laborales agrícolas y pecuarias. Modalidades integradas por docentes escogidos según su perfil y formación profesional, que laboran en el nivel de educación media técnica agropecuaria en la institución ${ }^{37}$. En este sentido, el fin teleológico del objetivo específico le da a la interculturalidad una lógica de complementariedad con saberes y conocimientos propios, al currículo oficial. Es una actitud proactiva hacia la consolidación de un plan de vida de buen vivir para los egresados, más allá de realizarse como bachilleres técnicos.

De otra parte, los directivos de la institución Thelma Rosa Arévalo, seleccionaron un equipo de maestros considerados de alto empoderamiento con su comunidad educativa para participar cooperativamente con el proceso investigativo. Se realizaron dos encuentros en las instalaciones de la Universidad del Magdalena, teniendo como propósito socializar la categoría epistémica "currículo intercultural". Esbozándose de manera amplia, con sentido didáctico, la participación reflexiva de los convocados fue prolífica desde sus voces. Se planteó elaborar una espina de pescado cuyos componentes de destello de

35 Víctor D. Bonilla, Orlando Fals Borda, Gonzalo Castillo y Augusto Libreros Causa popular, ciencia popular. Una metodología del conocimiento científico a través de la acción (Bogotá: Publicaciones de la Rosca, 1972), 61.

36 "Proyecto Educativo Institucional" (Varela-Magdalena, Colombia), Archivo Institución Educativa Departamental Thelma Rosa Arévalo, 118.

37 Ibíd., 40. 

para la paz y la justicia territorial en el Caribe colombiano, 2018-2019

reflexión fueron: currículo oficial, currículo intercultural, producción, reproducción desde la experiencia de vida y profesional de los maestros participantes.

Lo particular de este currículo es el énfasis que hacemos en la organización de contenidos y la descripción de saberes y conocimientos de la comunidad [...]. Con ello, también buscamos instar a los maestros a desarrollar una educación con identidad cultural desde la escuela, primero planificando y desarrollando sus prácticas pedagógicas escolares [...] a partir de saberes y conocimiento propio y segundo, complementando la formación del estudiante con los saberes y conocimientos llamados "universales". 38

De igual modo, la alianza estratégica entre la Universidad del Magdalena a través de su grupo de investigación Calidad Educativa en un Mundo Plural (CEMPLU) y la Institución Educativa Departamental Thelma Rosa Arévalo, logró concientizar a la masa crítica profesoral de la escuela intervenida. En este sentido, un grupo de profesores con título de maestría se dio a la tarea de organizar el primer conversatorio para recuperar la historia, valores e identidad cultural del corregimiento de Varela; el evento contó también con el apoyo de la Cooperativa de Productores de Banano del Magdalena (BANAFRUCOOP), uno de cuyos dirigentes afirmó:

Nosotros quisiéramos extender mucho más, de hecho como miembro de la junta Directiva me siento muy orgulloso de los asociados de la cooperativa porque ellos muy generosamente se han desprendido de recursos que eran para fortalecerlos a ellos para hacer esta gran labor social y educativa y es la de apoyarlos a ustedes. Nos preocupa mucho lo que ustedes acaban de decir y es el sentido de pertenencia de nuestra comunidad, de nuestros representantes y no es que queramos, somos muy aparte del tema politico y hemos sido siempre muy claros con ustedes en ese aspecto pero nos preocupa muchísimo que esto siga pasando en estos municipios que no progresan es por eso, por la falta de decisión, por no saber escoger personas a quienes realmente les preocupe el tema de la salud, de la educación, la situación de los colegios. ${ }^{39}$

Uno de los investigadores nutrió la dinámica del conversatorio invocando al filósofo y profesor mexicano Luis Villoro, quien afirma:

Un pueblo comienza a reconocerse cuando descubre las creencias, actitudes y proyectos básicos que prestan una unidad a sus diversas manifestaciones culturales y dan respuesta a sus necesidades reales. La identidad de un pueblo no puede describirse, por lo tanto, por las características que lo singularizan frente a los demás, sino por la manera concreta como se expresan, en una situación dada, sus

38 Fundación para la Educación en Contextos de Multilinguismo y Pluriculturalidad, Currículo comunitario local Yurakaré. Comunidad de Nueva Galilea (Cochabamba: Funproeib-Andes, 2011), 17.

39 Entrevista a representante de la Cooperativa de Productores de Banano del Magdalena (BANAFRUCOOP), Varela (Magdalena), 30 de abril de 2019. 
necesidades y deseos y se manifiestan sus proyectos, sean éstos exclusivos o no de ese pueblo. ${ }^{40}$

La experiencia surgida del proceso investigativo lleva a revisar el PEI y a reafirmar que lo allí planteado adquiere fuerza pedagógica, puesto que el modelo pedagógico ha sido trazado desde un enfoque constructivista, en el cual el currículo intercultural puede generar espacios favorables para los aprendizajes, teniendo como referente los saberes y conocimientos locales. Aspectos mediados por la cooperación recíproca entre estudiantes- maestros, padres-hijos y padres-maestros.

\section{CONCLUSIONES}

La Investigación Acción Cooperativa (IAC) desarrollada entre el grupo CEMPLU y la Institución Educativa Thelma Rosa Arévalo del corregimiento de Varela del municipio de Zona Bananera (Magdalena), contribuyó a estrechar lazos de amistad, academia y reflexión acerca de lo que produce en las personas el arraigo al territorio y los sentimientos que emergen al hacerse parte de él, en lo que se podría llamar identidad, punto de partida para empoderar la categoría potente del estudio, "Currículo intercultural". En este sentido, el grupo CEMPLU capacitó a tres grupos de maestros de la institución mencionada. Uno en las instalaciones de la Universidad del Magdalena (directivos), otro en la jornada de la mañana (primaria) y el tercero en la jornada de la tarde (bachillerato), estos dos últimos en las instalaciones del plantel educativo (IED Thelma Rosa Arévalo).

La intervención científica desarrollada de manera cooperativa arrojó las siguientes conclusiones:

1. ${ }^{\text {- }}$ La comunidad educativa de la Institución Educativa Thelma Rosa Arévalo y, en general, la comunidad del corregimiento de Varela, sigue siendo víctima del conflicto colombiano. Hoy se viene dando la restitución de tierras y, con ello, la aparición de bandas emergentes que se oponen a dicha restauración. Asimismo, se está dando la mora en los procesos judiciales contra las compañías exportadoras de banano, patrocinadoras de acciones paramilitares, situación que genera zozobra y miedo en el ambiente escolar.

2. ${ }^{a}-\quad$ La población de Varela fue víctima de desplazamiento forzado en el año 2000, a raíz de la masacre de cuatro campesinos. Hoy se ha dado un retorno al territorio de manera moderada. Los niños que asisten al plantel escolar no son conscientes de lo sufrido por sus padres y abuelos y se muestran indiferentes ante los hechos que se les narran. Son estudiantes de mentalidad globalizada y homogénea, adaptados al currículo oficial ofrecido por su escuela.

40 Luis Villoro, “Sobre la identidad de los pueblos", en Estado plural, pluralidad de culturas (México: UNAM/Paidós, 1998 ), 64. 
3. ${ }^{\text {era }}$ La institución promueve la categoría paz como principio de convivencia escolar, con el objeto de manifestar buena disciplina en la convivencia con sus pares y maestros. No se dimensiona desde el ámbito socio-histórico de la localidad y la globalidad.

4. ${ }^{\text {a- }} \quad$ La población de Varela no se identifica como afrodescendiente; para esta comunidad, afros son las personas de Sevilla, Guacamayal, Soplador, comunidades que hacían los cortes cuando operaba la United Fruit. Los varelenses ${ }^{41}$ se consideran buenos comerciantes, provenientes de otras regiones y países como Italia (el napolitano) ${ }^{42}$.

5. ${ }^{a}-\quad$ El acercamiento que hace la institución de manera casual a las dimensiones de la categoría interculturalidad, se pone de manifiesto a través de un proyecto de investigación del Programa Ciclón-Gobernación del Magdalena, cuyo tema fue el rescate de la memoria histórica de Varela, sus mitos y creencias ancestrales. Los maestros son conscientes del patrimonio cultural del contexto, pero manifiestan que hay que cumplir con lo ordenado por el Ministerio de Educación Nacional.

6. ․ - El Proyecto Educativo Institucional se presenta como un documento extremadamente técnico, sin espacios donde se realicen comprensiones que lleven a tomar decisiones y reconstrucciones pedagógicas, didácticas y epistemológicas que soporten el desarrollo pleno de la comunidad educativa.

La investigación arrojó, como nuevo conocimiento para las ciencias de la educación, afirmar que el currículo intercultural exige de las comunidades educativas locales y de sus miembros el conocerse "a sí mismos". Sin este conocimiento elemental no se concibe la impronta que marca la identidad de las personas. Cada ser humano posee una personalidad única, la cual desarrolla afrontando las dinámicas sociales de la cotidianidad, en un hilo del tiempo, el cual reclama cambios en sus comportamientos ${ }^{43}$.

La anterior reflexión epistémica surge tras haber llevado el proceso investigativo a la comunidad del corregimiento de Varela y su institución educativa. Esta población no ha logrado consolidarse con una identidad propia, debido a su itinerancia como comerciantes de algodón y banano o a su desplazamiento forzado por parte de los actores armados del conflicto colombiano. Es necesario, entonces, que las partes cooperantes del proceso investigativo generen espacios de empoderamiento de los habitantes de sentirse y creerse varelense y con ello iniciar la revalorización de sus tradiciones, costumbres y saberes legados. Para lograr lo anterior se sugiere reiniciar la Investigación Acción Cooperativa (IAC) en un segundo nivel óntico-epistémico en el espiral metodológico.

41 Gentilicio de las personas oriundas del corregimiento de Varela (Magdalena).

42 Personaje emblemático del corregimiento de Varela (Magdalena)

43 Amin Maalouf, Identidades asesinas (Madrid: Alianza Editorial, 1999), 9. 


\section{REFERENCIAS}

Arenas, Vivian, Karina Mora Wilches y Lisney Medina Mendoza. "Familia y escuela como entidades fundamentales en la construcción de paz". AIBI revista de investigación, administración e ingeniería 7, n.․ 51 (2019): 24-31.

Bartolomé, Margarita. "La investigación cooperativa". En Problemas y métodos de investigación en educación personalizada, coordinado por Víctor García Hoz. Madrid: Rialp, 1994.

Bolaños Motta, José Ignacio. “Aulas multiculturales y aulas de paz. Dos propuestas para un país en postconflicto". Revista Historia de la Educación Latinoamericana 20, n.․ 31 (2018). Doi: 10.19053/01227238.8563. https://revistas.uptc.edu.co/revistas/index.php/historia_educacion_latinamerican/issue/view/558

Bonilla, Víctor D., Orlando Fals Borda, Gonzalo Castillo y Augusto Libreros. Causa popular, ciencia popular. Una metodología del conocimiento científico a través de la acción. Bogotá: Publicaciones de la Rosca, 1972.

Cañón Flórez, Lilia, Myriam Monroy González, y Javier Salcedo Casallas. “Experiencias pedagógicas y voz multicultural. Pasos y palabras en historias de vida de maestra afrodescendiente". Revista Historia de la Educación Latinoamericana 18, n.ํ 27 (2016). Doi: 10.19053/01227238.5527. https://revistas.uptc.edu.co/index.php/historia_educacion_latinamerican/article/view/5527/4622

Castillo Guzmán, Elizabeth y Sandra Patricia Guido Guevara,. “La interculturalidad: ¿principio o fin de la utopía?". Revista Colombiana de Educación, n. ${ }^{\circ} 69$ (2015): 17-43.

Congreso de Colombia. Ley 115/1994, 8 de febrero, Ley General de la Educación. Diario Oficial n. ${ }^{\circ}$ 41.214. http://www.secretariasenado.gov.co/senado/basedoc/ley_0115_1994.html

Córdova, Gavina. "Diálogo sobre interculturalidad". ISEES: Inclusión Social y Equidad en la Educación Superior n. 7 (2010): 97-112.

De Oña Cots, José Manuel y Emilio Andrés García Gálvez. "Proyecto Escuela: Espacio de Paz. Reflexiones sobre una Experiencia en un Centro Educativo". REICE. Revista Iberoamericana sobre Calidad, Eficacia y Cambio en Educación 14, n. ${ }^{\circ}$ (2016): 115-131.

De Souza Silva, José. "La pedagogía de la felicidad en una educación para la vida". En Pedagogías decoloniales: Prácticas insurgentes de resistir, (re)existir y (re)vivir, tomo I, editado por Catherine Walsh. Quito: Ediciones Abya Yala, 2018.

Entrevista a representante de la Cooperativa de Productores de Banano del Magdalena (BANAFRUCOOP), Varela (Magdalena), 30 de abril de 2019.

Fonseca Pérez, Juan José y Michel Enrique Gamboa Graus. “Aspectos teóricos sobre el diseño curricular y sus particularidades en las Ciencias. Boletín Virtual 6, n. 3 (2017): 83-112.

García Araque, Fabio Alberto. “La etnoeducación como elemento fundamental en las comunidades afrocolombianas". Diálogos sobre Educación 8, n. 15 (2017): 1-21.

González Monroy, Luis Alfredo e Iván Manuel Sánchez Fontalvo. Interpelaciones interculturales en el currículo de las escuelas del retorno. Referentes para vislumbrar el posconflicto en Colombia. Santa Marta, Colombia: Editorial Unimagdalena, 2016.

González Monroy, Luis Alfredo. "Escuelas del retorno después del desplazamiento forzado en Colombia. Significados y sentidos dialógicos de un currículo intercultural". Tesis de doctorado en Ciencias de la Educación-RUDECOLOMBIA, Universidad del Magdalena, 2015.

Higuera Aguirre, Édison Francisco y Néstor Castillo Mantuano. “La interculturalidad como desafío para la educación ecuatoriana". Sophia: Colección de Filosofía de la Educación n. ${ }^{\circ} 18$ (2015): 147-162.

Lehmann, David. "Convergencias y divergencias en la educación superior intercultural en México". Revista Mexicana de Ciencias Políticas y Sociales LX, n. ${ }^{\circ} 223$ (2015): 133-170.

Maalouf, Amín. Identidades asesinas. Madrid: Alianza Editorial, 1999.

Meneses Copete, Yeison. "La etnoeducación afrocolombiana: conceptos, trabas, patriarcado y sexismo. A propósito de los 20 años de la Ley General de Educación 115 de 1994". Revista Historia de la Educación Latinoamericana 18, n. ${ }^{\circ} 27$ (2016): 35-66. https://doi. org/10.19053/01227238.5508

Oja, Sharon Nodie y Gerald J. Pine. Collaborative Action Research: A Two Year Study of Teachers' Stages 
of Development and School Contexts. Executive Summary. New Hampshire University, 1983.

Olivera Rodríguez, Inés. “Las potencialidades del proyecto educativo de la Universidad Veracruzana Intercultural: una crítica al desarrollo desde la noción del Buen vivir". Revista de la Educación Superior 46, n. 181 (2017): 19-35. https://www.redalyc.org/articulo.oa?id=60450097002

Pérez Dávila, Fredy Luis. "Políticas educativas en Colombia: en busca de la calidad". Actualidades Pedagógicas n.. 71 (2018): 193-213. Doi: http://dx.doi.org/10.19052/ap.4430

"Proyecto Educativo Institucional". Varela-Magdalena (Colombia). Archivo Institución Educativa Departamental Thelma Rosa Arévalo.

Sánchez Castellón, Emilce Beatriz. "Etnoeducación y prácticas interculturales de saberes otros". Utopía y Praxis Latinoamericana 23, n.. 83 (2018): 166-181. https://www.redalyc.org/articulo. oa?id=27957772015

Sánchez Fontalvo, Iván Manuel. La interculturalidad desde la perspectiva de la inclusión socioeducativa. Santa Marta: Editorial Unimagdalena, 2017.

Fundación para la Educación en Contextos de Multilinguismo y Pluriculturalidad. Currículo comunitario local Yurakaré. Comunidad de Nueva Galilea. Cochabamba: Funproeib-Andes, 2011.

Tostado Reyes, Elizabeth y Saúl Alejandro García. “Convivencia con conflicto y sin violencia en la escuela: la prevención como estrategia de paz". Ra Ximhai 11, n. ${ }^{\circ}$ (2015): 19-32. //www. redalyc.org/articulo.oa?id $=46139401001$

Triviño, Celina y Luisa Amézquita. "Reflexiones desde la universidad sobre la educación en derechos humanos y para la paz". Revista Historia de la Educación Latinoamericana 20, n. ${ }^{\circ}$ 31 (2018). Doi: 10.19053/01227238.8564. https://revistas.uptc.edu.co/revistas/index.php/ historia_educacion_latinamerican/issue/view/558

Tubino, Fidel. "El interculturalismo y los Estados nacionales". Conferencia magistral. Foro latinoamericano sobre interculturalidad, ciudadanía y educación. Cuetzalán, México: FLAPE, 2004.

Villoro, Luis. "Sobre la identidad de los pueblos". En Estado plural, pluralidad de culturas. México: UNAM/Paidós, 1998.

Walsh Catherine. "Pedagogías decoloniales. Prácticas insurgentes de resistir, (re) existir y (re) vivir". Tomo 1. Quito: Editorial ABYA YALA, 2018. Recuperado en https://www.traficantes. net/libros/pedagog\%C3\%ADas-decoloniales-tomo-1

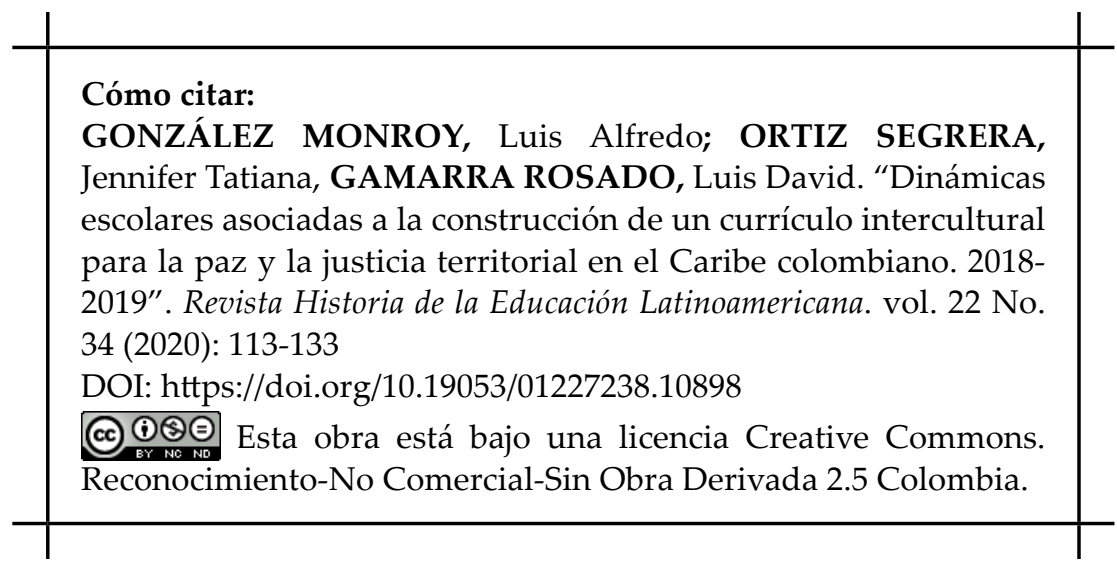

